\title{
Integrated Flight Dynamics and Aeroelasticity of Flexible Aircraft with Application to Swept Flying Wings
}

\author{
Robert J. S. Simpson* and Rafael Palacios ${ }^{\dagger}$ \\ Imperial College, London, SW7 2AZ, United Kingdom
}

The dynamics of flexible, swept flying wing (SFW) aircraft are described by a set of nonlinear, multi-disciplinary equations of motion. Aircraft structures are modeled using a geometrically-exact composite beam model which can, in general, capture large dynamic deformations and the interaction between rigid-body and elastic degrees-offreedom. In addition, an implementation of the unsteady vortex-lattice method capable of handling arbitrary kinematics is used to capture the unsteady, three-dimensional flow-field around the aircraft as it deforms. Linearization of this coupled nonlinear description, which can in general be around a nonlinear equilibrium, is performed to yield linear time-invariant state-space models. Verification of aeroelastic stability analyses using these models is carried out. Subsequently, a set of SFW models are developed and the dynamic stability characteristics of these aircraft are investigated for a range of flight velocities and vehicle parameters.

\section{Nomenclature}

$A \quad$ state-space system matrix / sectional area, $\mathrm{m}^{2}$

$\mathcal{A}$ aerodynamic influence coefficient matrix (downwash)

$\mathcal{A}_{\boldsymbol{U}} \quad$ aerodynamic influence coefficient matrix (velocity)

$B \quad$ state-space input matrix

$C \quad$ constant sparse matrix / state-space output matrix

$\mathcal{C} \quad$ tangent damping matrix

\footnotetext{
${ }^{*}$ Graduate Student, Department of Aeronautics, 363A Roderic Hill Building; r.simpson11@imperial.ac.uk. AIAA Student Member.

${ }^{\dagger}$ Reader, Department of Aeronautics, 355 Roderic Hill Building; r.palacios@imperial.ac.uk. AIAA Member.
} 


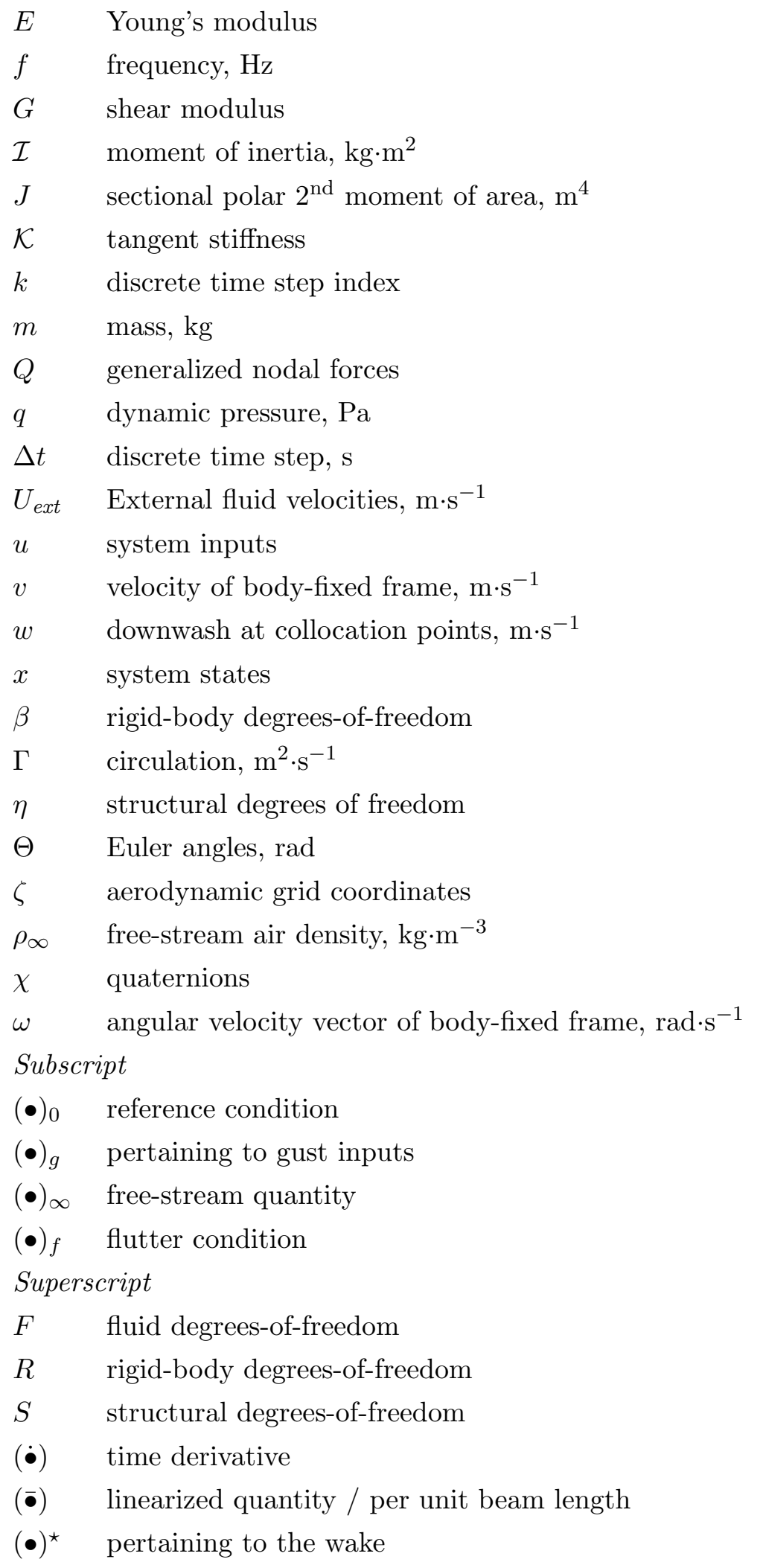




\section{Introduction}

The trend for ever more lightweight, slender aero-structures is pushing the design of nextgeneration aircraft towards unprecedented levels of flexibility. A consequence of this is that active aeroelastic control will be employed more often for stability augmentation and gustload alleviation, and is likely to be an enabling technology for future designs. This has forced engineers to develop multi-disciplinary computational tools for early-stage and control system design that can predict the interaction of aircraft elastic response, rigid-body motion, and aerodynamic forcing. ${ }^{1,2,3,4,5,6}$ In addition to this, several flight test programs ${ }^{7,8}$ have been developed recently that specifically target the phenomenon of aeroelastic/rigid-body coupling with the intention of developing active aeroelastic control systems for stability augmentation and disturbance rejection. Most saliently, the intended handover of the Lockheed-developed X-56 Multi-Utility Technology Testbed (MUTT) to NASA has created a focal point for public domain research into flexible aircraft flight dynamics (FAFD) and control. ${ }^{8,9,10}$

As such, the goals of this work are to present a relatively simple test case, referred to as the Swept Flying Wing (SFW), useful for investigating the control problems associated with platforms similar to the X-56 test aircraft. Firstly, the analysis methodology used in this work is described in Section II. Following this, verification of the static and dynamic aeroelastic analyses are presented in Section III. Finally, the development of the SFW test case is presented with stability analysis of the unconstrained aeroelastic response of the aircraft in Section IV. In the future it is hoped that this computational model will be useful for demonstrating active aeroelastic control technologies for stabilization and disturbance rejection during simulations of the aircraft dynamics.

\section{Methodology}

In this section we present a flexible aircraft flight dynamics model based on coupling a nonlinear composite beam model with the unsteady vortex-lattice method (UVLM). Following this we present a linearization of the coupled model, and the re-casting of its underlying equations into state-space form. These methods are the basis for the software implementation used in this work, referred to as Simulation of High-Aspect-Ratio Planes in Python (SHARPy).

\section{II.A. Nonlinear Flexible Aircraft Dynamics}

A geometrically-exact composite beam model based on the formulations by Hodges ${ }^{11}$ and Géradin \& Cardona ${ }^{12}$ is used to capture the dynamics of flexible, unconstrained structures subject to large (geometrically-nonlinear) deformations and nonlinear rigid-body motion. 
A moving body-fixed reference system is used to track elastic deformations and rigid-body motion of the structure. The resulting equations of motion (EoM) that describe a moving flexible body are ${ }^{13}$

$$
\mathcal{M}(\eta)\left\{\begin{array}{l}
\ddot{\eta} \\
\dot{\beta}
\end{array}\right\}+Q_{g y r}(\eta, \dot{\eta}, \beta)+Q_{s t i f}(\eta)=Q_{e x t}(\eta, \dot{\eta}, \beta, \chi)
$$

where the structural degrees of freedom, $\eta$, are given in terms of nodal displacements and finite rotations (parameterized using the Cartesian rotation vector) ${ }^{12}$ in a 2 -noded finiteelement beam discretization. The rigid-body states, $\beta$, include the translational and angular velocities of the origin of the body-fixed reference frame. These are denoted $v_{a}$ and $\omega_{a}$ respectively. The system dynamics are coupled through the tangent mass matrix, $\mathcal{M}(\eta)$, and the discrete gyroscopic and external forces, $Q_{g y r}$ and $Q_{e x t}$, respectively. Computation of the discrete elastic forces, $Q_{\text {stif }}$, depend upon the elastic degrees-of-freedom, $\eta$. The structural and rigid-body components (denoted by superscripts $S$ and $R$, respectively) of the gyroscopic, elastic and external forces can be identified as

$$
Q_{g y r}=\left\{\begin{array}{c}
Q_{g y r}^{S} \\
Q_{g y r}^{R}
\end{array}\right\}, Q_{s t i f}=\left\{\begin{array}{c}
Q_{s t i f}^{S} \\
0
\end{array}\right\}, \text { and } Q_{e x t}=\left\{\begin{array}{c}
Q_{e x t}^{S} \\
Q_{e x t}^{R}
\end{array}\right\}
$$

respectively. A detailed description of the various terms in Eq. (1) can be found in previous work by one of the authors. ${ }^{13}$ Finally, the orientation of the body-fixed reference frame with respect to an inertial frame is parameterized using quaternions, denoted by $\chi$, and computed by solving an extra set of attitude EoM. ${ }^{14,15}$ Together with the Eq. (1) this completes the geometrically nonlinear description of the aircraft structural dynamics and nonlinear rigidbody motion.

Assuming coincident spanwise discretizations, an aerodynamic lattice $\zeta$ is constructed that represents the aircraft lifting surfaces. From this discretization, the $3-\mathrm{D}$, geometricallynonlinear, unsteady aerodynamic loading is obtained using the unsteady vortex-lattice method. In the UVLM, quadrilateral vortex ring elements are used to discretize both lifting surfaces and their wakes. Each surface (bound) vortex ring has an associated circulation strength, $\Gamma$, and a collocation point at which the non-penetration boundary condition is satisfied. The Kutta condition and Joukowski hypothesis ${ }^{16}$ are approximately satisfied by shedding wake vortex rings from the trailing-edge of each surface at every time step. A first-order, explicit time-stepping scheme is employed. ${ }^{4,17,18}$ This results in a time-varying, discrete-time system 
of equations, written for time step $k+1$, of the form

$$
\begin{gathered}
\mathcal{A}(\zeta) \Gamma^{k+1}+\mathcal{A}^{\star}\left(\zeta, \zeta^{\star}\right) \Gamma^{\star^{k+1}}+w^{k+1}=0 \\
\Gamma^{\star^{k+1}}=C_{\Gamma} \Gamma^{k}+C_{\Gamma}^{\star} \Gamma^{\star^{k}} \\
\zeta^{\star^{k+1}}-C_{\zeta} \zeta^{k+1}=C_{\zeta}^{\star} \zeta^{\star^{k}}+\Delta t\left(\mathcal{A}_{\boldsymbol{U}} \Gamma^{k}+\mathcal{A}_{\boldsymbol{U}}^{\star} \Gamma^{\star^{k}}\right)+\Delta t U_{e x t}^{\star^{k}},
\end{gathered}
$$

where Eq.(3) enforces the non-penetration boundary condition on the aircraft geometry, and Eqs. (4) \& (5) propagate the wake circulation strength and wake geometry, respectively, through time. Note that the ()$^{\star}$ superscript denotes an entity corresponding to the aircraft wake, for example the lattice geometry of the free shear-layer, $\zeta^{\star}$, or the corresponding vortex ring strengths, $\Gamma^{\star}$. A more detailed description of the terms in (3) - (5) can be found in previous work by the authors. ${ }^{19}$

The circulation distribution on the deformed aircraft is then post-processed to obtain the aerodynamic loading which is then mapped back onto the structural nodes. Thus the flexiblebody dynamics and aerodynamics models are coupled to form a nonlinear aeroelastic model of the dynamics of flexible aircraft. In the presence of geometrically nonlinear deformations and complex kinematics all components of the aerodynamic forces must be captured when post-processing the aircraft states to obtain aerodynamic load distributions. ${ }^{20,21}$

\section{II.B. Linearization}

The structural dynamics are linearized around reference condition $\left(\eta_{0}, \dot{\eta}_{0}, \beta_{0}, \chi_{0}\right)$ and small changes from this state are represented with over-bars, that is, $(\bar{\eta}, \dot{\bar{\eta}}, \bar{\beta}, \bar{\chi})$. The linearized (incremental) form of Eq. (1) around the reference state has the form ${ }^{13}$

$$
\mathcal{M}\left(\eta_{0}\right)\left\{\begin{array}{c}
\ddot{\bar{\eta}} \\
\dot{\bar{\beta}}
\end{array}\right\}+\mathcal{C}\left(\eta_{0}, \dot{\eta}_{0}, \beta_{0}\right)\left\{\begin{array}{l}
\dot{\bar{\eta}} \\
\bar{\beta}
\end{array}\right\}+\mathcal{K}\left(\eta_{0}, \dot{\eta}_{0}, \beta_{0}\right)\left\{\begin{array}{l}
\bar{\eta} \\
0
\end{array}\right\}=\bar{Q}_{e x t}(\bar{\eta}, \dot{\bar{\eta}}, \bar{\beta}, \bar{\Theta})
$$

where the constant tangent damping and stiffness matrices $\mathcal{C}$ and $\mathcal{K}$, respectively, are obtained through direct linearization of the discretized forces in Eq. (1). Note that Euler angles, $\Theta$, are preferred in the linear analysis to describe variations of the aircraft orientation. ${ }^{22}$ Projection of the linear EoM on the vibration modes of the unconstrained aircraft can further simplify the structural representation of the vehicle dynamics. This linear, secondorder set of ODEs is then discretized in time using the Newmark- $\beta$ scheme and cast in state-space form and coupled with a linearized UVLM aerodynamics model.

A linearized form of the aerodynamics model is constructed using the model developed by Murua et al. ${ }^{23}$ and based on the description by Hall. ${ }^{24}$ To obtain the state-space form of the UVLM, the governing equations are linearized on a frozen aerodynamic geometry around the 
aircraft trim condition, which may include large wing deformations and a non-planar wake. Therefore, the system of Eqs. (3)-(5) is reduced to

$$
\begin{gathered}
\mathcal{A}\left(\zeta_{0}\right) \bar{\Gamma}^{k+1}+\mathcal{A}^{\star}\left(\zeta_{0}, \zeta_{0}^{\star}\right) \bar{\Gamma}^{\star^{n+1}}+\bar{w}^{k+1}=0, \\
\bar{\Gamma}^{\star^{k+1}}=C_{\Gamma} \bar{\Gamma}^{k}+C_{\Gamma}^{\star} \bar{\Gamma}^{\star^{k}}
\end{gathered}
$$

where the over-bars again represent increments on the states about which the aircraft is linearized. The aerodynamic equations above are coupled with the linear structural Eqs. (6) and cast in the discrete-time state-space form

$$
\begin{aligned}
x(k+1) & =A x(k)+B u(k)+B_{g} u_{g}(k), \\
y(k) & =C x(k),
\end{aligned}
$$

where $u$ are the actuator inputs and subscript- $g$ terms correspond to gust inputs. The state vector that completely determines the linear system is

$$
x:=\left(x_{F} ; x_{S} ; x_{R}\right),
$$

where

$$
\begin{aligned}
& x_{F}:=\left(\bar{\Gamma} ; \bar{\Gamma}^{\star} ; \dot{\bar{\Gamma}}\right), \\
& x_{S}:=(\bar{\eta} ; \dot{\bar{\eta}}) \\
& x_{R}:=(\bar{\beta} ; \bar{\Theta})
\end{aligned}
$$

Note that the time-rate-of-change of the bound vortex ring strengths, $\dot{\bar{\Gamma}}$, is included in the state vector as it is required for the computation of unsteady aerodynamic loads. ${ }^{23}$ This set of coupled, linear EoM now form a suitable basis for stability analyses, model reduction, and control syntheses.

\section{Verification of aeroelastic analyses}

Divergence and flutter of swept wings was investigated numerically and experimentally by Ricketts and Doggett. ${ }^{25}$ In their experiments wind-tunnel models of forward-swept, isotropic, cantilever wings (with the structure being a flat plate) were subjected to a range of dynamic pressures, in some cases up to their divergence dynamic pressure, $q_{D}$. For some of the wing models flutter occurred prior to the onset of divergence - this was also reported and predicted numerically in their work. The wing models are described in the planform sketches of Figure 1 and structural properties of Table 1. This set of tests allows a benchmarking of 
the aeroelastic analysis in SHARPy - an example finite element model is shown in Figure 2 for the wing with 15 degrees forward sweep.

The natural modes of vibration of the wing models were calculated using SHARPy and the resulting modal frequencies are compared to published values in Table 2. The first two modes are $1^{\text {st }}$ and $2^{\text {nd }}$ flapwise bending, and the third is $1^{\text {st }}$ torsion. The small difference between SHARPy and the reference data comes from the beam approximation in this relatively short-aspect ratio wing. This difference is similar in magnitude to the computations in the reference, which were made using plate finite-elements.
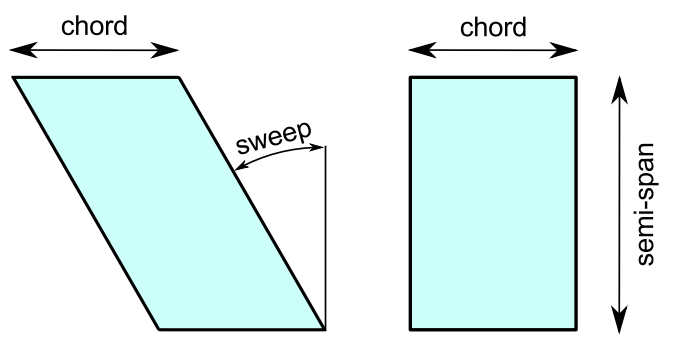

Figure 1. Planforms of the family of swept wings from Ricketts and Doggett. ${ }^{25}$

\begin{tabular}{ll}
\hline \hline Chord & $0.127 \mathrm{~m}$ \\
Semi-span & $0.508 \mathrm{~m}$ \\
Thickness & $0.00229 \mathrm{~m}$ \\
Density & $2712 \mathrm{~kg} \cdot \mathrm{m}^{3}$ \\
Shear Modulus & $26 \mathrm{GPa}$ \\
Poisson's ratio & 0.32 \\
\hline \hline
\end{tabular}

Table 1. Ricketts and Doggett's ${ }^{25}$ wing properties.

\begin{tabular}{c||c|c|c||c|c|c||c|c|c}
\hline \hline \multicolumn{1}{c||}{ Sweep } & \multicolumn{3}{c||}{ Ref. experimental } & \multicolumn{3}{c||}{ Ref. computational } & \multicolumn{4}{c}{ SHARPy } \\
(deg) & $f_{1}$ & $f_{2}$ & $f_{3}$ & $f_{1}$ & $f_{2}$ & $f_{3}$ & $f_{1}$ & $f_{2}$ & $f_{3}$ \\
\hline 0 & 7.0 & 43.8 & 59.8 & 7.4 & 46.3 & 59.8 & 7.21 & 45.4 & 55.0 \\
-7.5 & 6.9 & 42.8 & 57.2 & 7.3 & 45.5 & 60.0 & 7.09 & 44.7 & 55.0 \\
-15 & 6.6 & 41.2 & 56.4 & 7.0 & 43.2 & 60.4 & 6.73 & 42.4 & 55.0 \\
-30 & 5.5 & 33.4 & 57.4 & 5.8 & 35.3 & 60.7 & 5.41 & 34.1 & 55.0 \\
\hline \hline
\end{tabular}

Table 2. The first three natural vibration frequencies of the Ricketts and Doggett ${ }^{25}$ wings (in $\mathrm{Hz}$ ) as a function of sweep angle.

\section{III.A. Divergence}

Divergence analysis was carried out in SHARPy by conducting static analysis of the aeroelastic bending response at different angles of attack and sub-critical dynamic pressures. The tip 


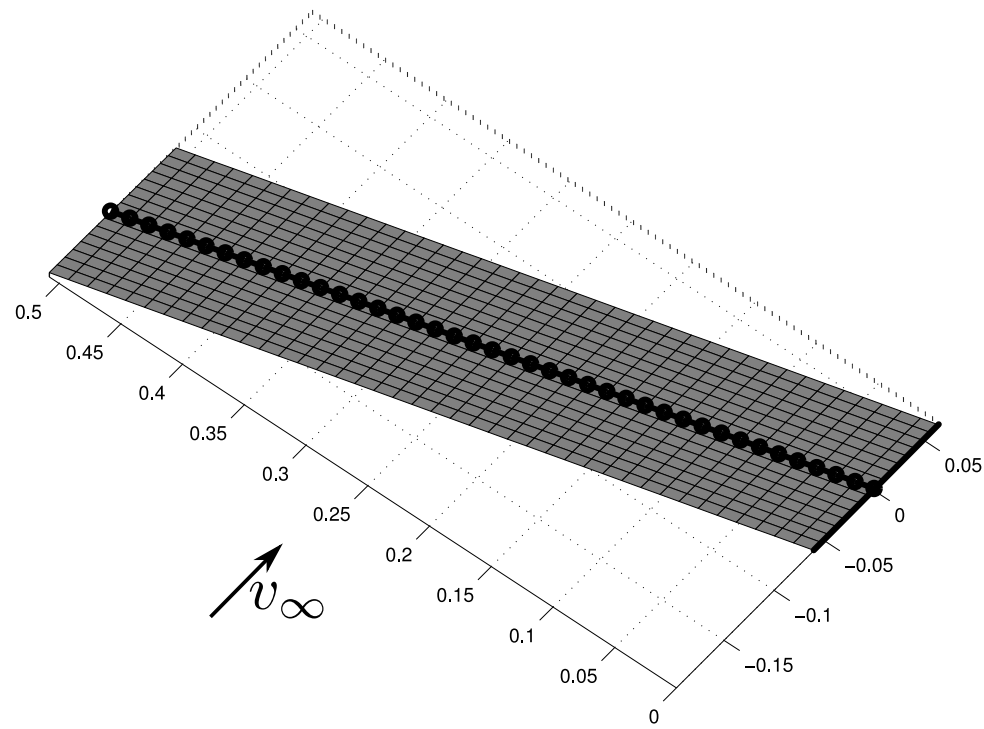

Figure 2. SHARPy finite-element model of the 15 degrees forward swept wing.

deflections arising from simulation of this parameter space were then used to extrapolate to the critical condition using a Divergence Index projection (DI). ${ }^{26}$ Results of this divergence analysis in SHARPy are compared with the experimental and computational work of Ricketts and Doggett in Figure 3. SHARPy compares well with experimental and numerical results in the reference at all sweep angles.

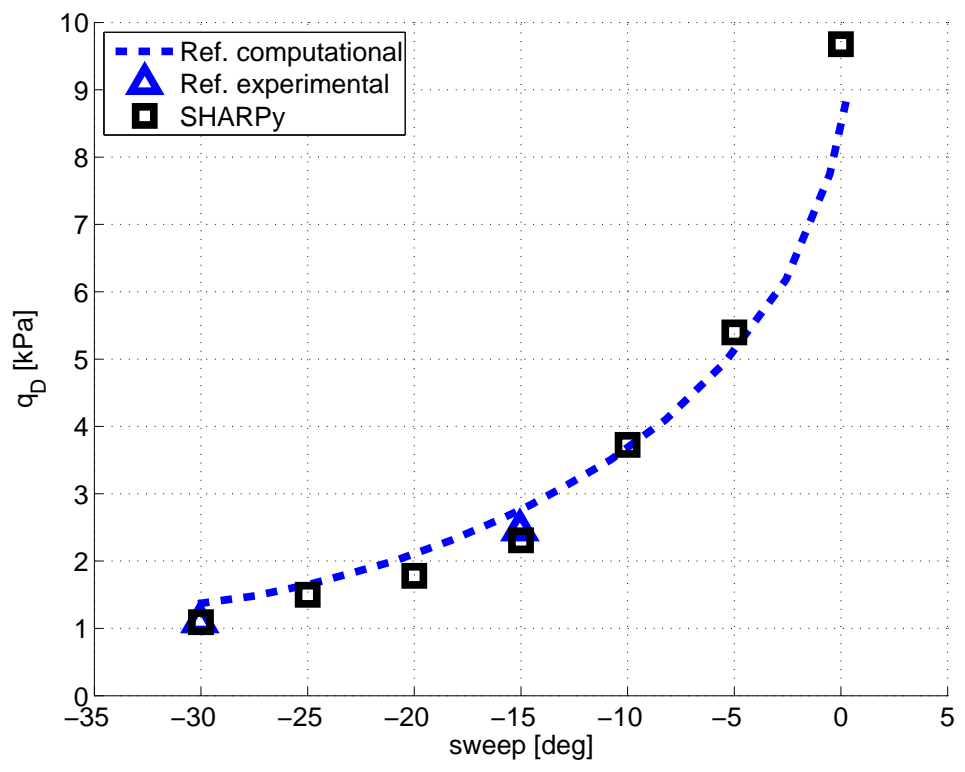

Figure 3. Comparison of divergence dynamic pressures calculated by SHARPy and those reported by Ricketts and Doggett ${ }^{25}$ for wings of varying sweep angle. 


\section{III.B. Flutter}

Flutter results were also obtained, using $\rho_{\infty}=1 \mathrm{~kg} \cdot \mathrm{m}^{-3}$, for sweep angles of $0,-7.5$ and -20 degrees. Note that for -20 deg sweep, divergence occurs before flutter, but the flutter onset can still be identified from linear stability analyses. The calculated flutter velocities and frequencies are given in Table 3. With zero sweep, a relatively low-frequency $(28.6 \mathrm{~Hz})$ flutter mechanism is observed (classical 1st bending / torsion flutter), whereas in the forward swept cases a high frequency $(>42 \mathrm{~Hz})$ but lower speed flutter is the critical dynamic instability (2nd bending / 1st torsion flutter). A comparison is made with the experimental and computational analysis of Ricketts and Doggett ${ }^{25}$ in Figure 4, which shows SHARPy to be in good, albeit slightly conservative, agreement with both analyses. This may be because the beam equations are discretized in time using a Newmark- $\beta$ scheme with $1 \%$ numerical damping.

\begin{tabular}{c|cc|cc|cc}
\hline \hline Model & \multicolumn{2}{|c|}{0 deg sweep } & \multicolumn{2}{c|}{-7.5 deg sweep } & \multicolumn{2}{c}{-20 deg sweep } \\
& $V_{f}\left[\mathrm{~m} \cdot \mathrm{s}^{-1}\right]$ & $\omega_{f}[\mathrm{~Hz}]$ & $V_{f}\left[\mathrm{~m} \cdot \mathrm{s}^{-1}\right]$ & $\omega_{f}[\mathrm{~Hz}]$ & $V_{f}\left[\mathrm{~m} \cdot \mathrm{s}^{-1}\right]$ & $\omega_{f}[\mathrm{~Hz}]$ \\
\hline SHARPy & 109.5 & 28.6 & 71.0 & 46.5 & 78.0 & 42.3 \\
\hline \hline
\end{tabular}

Table 3. Flutter results on forward swept wing $(\mathrm{AR}=4)$

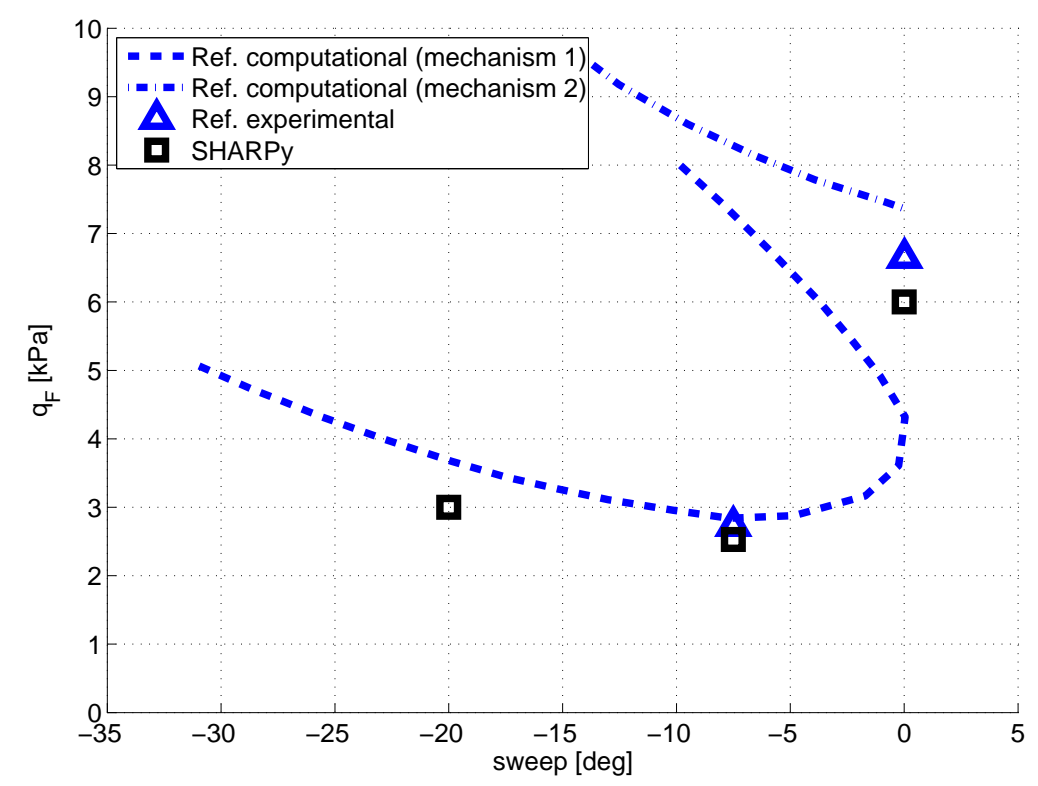

Figure 4. Comparison of flutter dynamic pressures $\left(\rho_{\infty}=1 \mathrm{~kg} \cdot \mathrm{m}^{-}\right)$calculated by SHARPy and those reported by Ricketts and Doggett. ${ }^{25}$ 


\section{SFW analysis}

\section{IV.A. Model definition}

A swept flying wing (SFW) model was created using the structural and aerodynamics models described in Sec. II and implemented in SHARPy. The geometry, mass distribution, and stiffness distribution of the model are symmetrical across the aircraft centre line and are described two symmetrical beam segments and a lattice geometry, shown in Figure 5. The geometry, mass and stiffness properties, which vary linearly from root to tip, are given in Table 4. In addition to the distributed mass of Table 4, two lumped masses of 40kg, with an associated pitch inertia of $5 \mathrm{~kg} \cdot \mathrm{m}^{2}$, were added to the model. These masses are located at $10 \%$ span on each wing, and are $0.1 \mathrm{~m}$ aft of the elastic axis. In the proceeding section the location of these masses, which notionally represent aircraft payload and propulsion systems, are varied to investigated any associated changes to the aircraft aeroelastic stability. Additionally, in Figure 5, the 2 inboard and 6 outboard are shown, which will be used for aeroelastic trim and control of the aircraft in subsequent work.

The first eight modes of the unconstrained SFW model are presented in Figure 6. The images show the elastic displacement modes relative to the body-fixed frame. Note that the node numbering starts from ten as the first nine modes are zero-frequency modes corresponding to the rigid-body dynamics degrees-of-freedom (6 velocities and 3 Euler angles). The low-frequency modes exhibit significant contributions from the rigid-body degrees of freedom, which are shown above each sub-figure. This description concludes the baseline SFW model.

\begin{tabular}{l|cc} 
& Root & Tip \\
\hline chord $[\mathrm{m}]$ & 1.15 & 0.65 \\
$\bar{m}\left[\mathrm{~kg} \cdot \mathrm{m}^{-1}\right]$ & 15 & 2 \\
$\overline{\mathcal{I}}_{1}[\mathrm{~kg} \cdot \mathrm{m}]$ & 0.5 & 0.1 \\
$E A[\mathrm{~N}]$ & $1.0 \times 10^{7}$ & $1.0 \times 10^{7}$ \\
$G J\left[\mathrm{~N} \cdot \mathrm{m}^{2}\right]$ & $7.5 \times 10^{4}$ & $3.0 \times 10^{4}$ \\
$E I_{2}\left[\mathrm{~N} \cdot \mathrm{m}^{2}\right]$ & $4.5 \times 10^{4}$ & $2.0 \times 10^{4}$ \\
$E I_{3}\left[\mathrm{~N} \cdot \mathrm{m}^{2}\right]$ & $2.4 \times 10^{6}$ & $1.0 \times 10^{6}$
\end{tabular}

Table 4. SFW geometry, mass and stiffness properties. Parameters vary linearly from root to tip.

\section{IV.B. Mass variations and stability analyses}

Firstly, the dynamic stability of the SFW model described in Section IV.A was investigated. From the similarity of this model to that of the X-56 it was expected that a coupled longitu- 
dinal rigid-body $/ 1^{\text {st }}$ symmetric bending mode, i.e. body-freedom flutter (BFF), would be the critical flutter mode. ${ }^{9}$ This behaviour is consistent with other recent work on the X-56 ${ }^{8}$ and other swept flying-wing configurations. ${ }^{27}$

To visualize the stability of the aircraft model throughout the flight envelope, linearized models were constructed for a range of velocities at sea-level air density $\left(\rho_{\infty}=1.225 \mathrm{~kg} \cdot \mathrm{m}^{-3}\right)$. Eigenvalues of the resulting state transfer matrices ( $A$ in Eq. (9)) were then used to construct root loci illustrating the evolution of the system dynamics with velocity. Figure 7 illustrates the dynamics of the baseline aircraft, described in Section IV.A, in this way. The shortperiod mode rises steadily in frequency and damping from close to the origin far into the left-hand plane. This is accompanied by the first bending mode (which starts at around $5 \mathrm{~Hz}$ ) dropping in frequency and showing a region of decreasing damping (becoming less stable) after $30 \mathrm{~m} \cdot \mathrm{s}^{-1}$. This root migrates into the right-hand plane at around $40 \mathrm{~m} \cdot \mathrm{s}^{-1}$, indicating BFF instability. The coalescence of modal frequencies and divergence of damping - one mode becoming unstable while the other, in this case the aircraft short period mode, rapidly becomes very highly damped - is analogous to the classical bend-twist flutter of unswept cantilever wings. The higher frequency modes of the aircraft remain in the left-hand plane, and are therefore stable, up to a velocity of $150 \mathrm{~m} \cdot \mathrm{s}^{-1}$.

To investigate the effect of mass distribution on the aircraft dynamic stability two modified SFW models were constructed. This was done by moving the lumped masses from $10 \%$ span (baseline case) to $20 \%$ span and $30 \%$ span. The chordwise offset of the masses are updated to maintain a distance of $0.1 \mathrm{~m}$ from the beam axis. The unconstrained natural mode frequencies and rigid-body mass properties of all three SFWs are compared in Table 5. Moving the concentrated masses outboard increases the rolling and yawing inertia, and reduces the pitch inertia.

Figure 8 shows the aeroelastic stability of the baseline model (black), 20\% span model (red) and $30 \%$ span model (green). In general the BFF mode velocity is increased as the lumped masses are moved outboard: $40 \mathrm{~m} \cdot \mathrm{s}^{-1}$ for the baseline case, $41 \mathrm{~m} \cdot \mathrm{s}^{-1}$ for the $20 \%$ span case, and $52 \mathrm{~m} \cdot \mathrm{s}^{-1}$ for the $30 \%$ span case. This has the side-effect of introducing a second, high frequency, instability in the $2^{\text {nd }}$ antisymmetric bending / torsion mode, which occurs at $150 \mathrm{~m} \cdot \mathrm{s}^{-1}$ and $14.3 \mathrm{~Hz}$ in the $20 \%$ span case. This effect is more pronounced for the $30 \%$ span model where the same mode becomes unstable, but at $120 \mathrm{~m} \cdot \mathrm{s}^{-1}$ and 12.45 $\mathrm{Hz}$.

\section{Conclusion}

A relatively simple swept-flying-wing (SFW) configuration, designed to replicate the stability problems typical of such aircraft, namely body-freedom-flutter (BFF) and higher fre- 


\begin{tabular}{l|ccc}
\hline \hline Baseline mode & Baseline & 20\% span & 30\% span \\
\hline $1 \mathrm{sfb}$ & 5.15 & 5.00 & 5.02 \\
$1 \mathrm{asfb}$ & 8.31 & 7.05 & 6.77 \\
2sfb & 15.8 & 16.8 & 13.9 \\
2asfb/1asT & 20.3 & 17.3 & 16.9 \\
1 sllb & 22.2 & 21.7 & 23.4 \\
$3 \mathrm{asfb} / 2 \mathrm{asT}$ & 23.7 & 22.9 & 24.9 \\
$1 \mathrm{sT}$ & 34.2 & 35.7 & 30.3 \\
$3 \mathrm{asT} / 3 \mathrm{asfb}$ & 35.4 & 37.7 & 41.1 \\
\hline$m_{\text {total }}[\mathrm{kg}]$ & 153.1 & 153.1 & 153.1 \\
CoG $[\% \mathrm{chord}]$ & 71.90 & 79.02 & 86.17 \\
$\mathcal{I}_{\text {roll }}\left[\mathrm{kg} \cdot \mathrm{m}^{2}\right]$ & 255.4 & 293.8 & 357.8 \\
$\mathcal{I}_{\text {pitch }}\left[\mathrm{kg} \cdot \mathrm{m}^{2}\right]$ & 28.18 & 24.35 & 22.42 \\
$\mathcal{I}_{\text {yaw }}\left[\mathrm{kg} \cdot \mathrm{m}^{2}\right]$ & 271.0 & 305.6 & 367.6 \\
\hline \hline
\end{tabular}

Table 5. Natural vibration frequencies (in $\mathrm{Hz}$ ) and rigid-body properties of the unconstrained SFW aircraft models. Mode descriptions are made using acronyms, for example, the first symmetric flapwise bending mode is denoted $1 \mathrm{sfb}$ and the first antisymmetric torsional mode is denoted 1asT. The CoG is expressed as the chordwise centre-line ordinate aft of the aircraft nose apex. The rigid-body inertias are calculated around the CoG.

quency antisymmetric bending / torsion flutter, has been developed. A methodology capable of modeling the complex coupled dynamics required to simulate these aircraft has also been presented. Aeroelastic verification of this implementation has been made with experimental and numerical tests of isotropic, swept, cantilever wings. In both divergence and flutter analyses there was close agreement between the implementation used in this work and the previous analyses. Unconstrained flutter analysis of a family of the SFW aircraft has shown the presence of a destabilizing mechanism in the $1^{\text {st }}$ symmetric bending mode, BFF. This flutter mechanism was delayed when mass was moved progressively outboard on the model. This introduced instability of the $2^{\text {nd }}$ antisymmetric bending / torsion mode at high velocities, however. It was shown that the spanwise distribution of mass can significantly affect the aeroelastic stability of swept flying wing aircraft, and in general moving mass outboard increases the velocity at which BFF occurs. In future work the SFW model will provide a basis for exploration of control system designs for stabilization and load-alleviation.

\section{Acknowledgments}

This work was supported by the UK Engineering and Physical Sciences Research Council under grant number EP/I014683/1. 


\section{References}

${ }^{1}$ van Schoor, M. C. and von Flotow, A. H., "Aeroelastic Characteristics of a Highly Flexible Aircraft," Journal of Aircraft, Vol. 27, No. 10, 1990, pp. 901 - 908.

${ }^{2}$ Shearer, C. M. and Cesnik, C. E., "Nonlinear flight dynamics of very flexible aircraft," Journal of Aircraft, Vol. 44, No. 5, 2007, pp. 1528 - 1545.

${ }^{3} J i a n$, Z. and Jinwu, X., "Nonlinear Aeroelastic Response of High-aspect-ratio Flexible Wings," Chinese Journal of Aeronautics, Vol. 22, No. 4, Aug. 2009, pp. 355 - 363.

${ }^{4}$ Wang, Z., Chen, P. C., Liu, D. D., and Mook, D. T., "Nonlinear-Aerodynamics/Nonlinear-Structure Interaction Methodology for a High-Altitude Long-Endurance Wing," Journal of aircraft, Vol. 47, No. 2, 2010 , pp. $556-566$.

${ }^{5} \mathrm{Su}, \mathrm{W}$. and Cesnik, C. E. S., "Dynamic Response of Highly Flexible Flying Wings," AIAA Journal, Vol. 49, No. 2, 2011, pp. $324-339$.

${ }^{6}$ Dillsaver, M. J., Cesnik, C. E. S., and Kolmanovsky, I. V., "Gust Response Sensitivity Characteristics of Very Flexible Aircraft," AIAA Atmospheric Flight Mechanics Conference, AIAA, Minneapolis, Minnesota, Aug. 2012.

${ }^{7}$ Cesnik, C. E., Senatore, P. J., Weihua, S., Atkins, E. M., and Shearer, C. M., "X-HALE: A Very Flexible Unmanned Aerial Vehicle for Nonlinear Aeroelastic Tests," AIAA journal, Vol. 50, No. 12, 2012, pp. $2820-2833$.

${ }^{8}$ Ryan, J. J., Bosworth, J. T., Burken, J. J., and Suh, P. M., "Current and Future Research in Active Control of Lightweight, Flexible Structures Using the X-56 Aircraft," 52nd Aerospace Sciences Meeting at SciTech, AIAA, National Harbour, MD, Jan. 2014.

${ }^{9}$ Bryson, D. E. and Alyanak, E. J., "Aeroelastic Modeling of the X-56A Using a Rapid Model Generator for Conceptual Design," 52nd Aerospace Sciences Meeting at SciTech, AIAA, National Harbour, MD, Jan. 2014.

${ }^{10}$ Hjartarson, A., Seiler, P. J., and Balas, G. J., "LPV aeroservoelastic control using the lpvtools toolbox," AIAA Atmospheric Flight Mechanics Conference, AIAA, Boston, MA, Aug. 2013.

${ }^{11}$ Hodges, D. H., "A mixed variational formulation based on exact intrinsic equations for dynamics of moving beams," International Journal of Solids and Structures, Vol. 26, No. 11, 1990, pp. 1253 - 1273.

${ }^{12}$ Géradin, M. and Cardona, A., Flexible Multibody Dynamics: A Finite Element Approach, John Wiley \& Sons Ltd, Chichester, UK, 2001.

${ }^{13}$ Hesse, H. and Palacios, R., "Consistent structural linearisation in flexible-body dynamics with large rigid-body motion," Computers \& Structures, Vol. 110 - 111, 2012, pp. 1 - 14.

${ }^{14}$ Stevens, B. L. and Lewis, F. L., Aircraft Control and Simulation, John Wiley \& Sons, Inc., New York, NY, USA, 1992.

${ }^{15}$ Murua, J., Hesse, H., Palacios, R., and Graham, J. M. R., "Stability and Open-Loop Dynamics of Very Flexible Aircraft Including Free-Wake Effects," 52nd AIAA/ASME/ASCE/AHS/ASC Structures, Structural Dynamics and Materials Conference, Denver, Colorado, April 2011.

${ }^{16}$ Morino, L. and Bernardini, G., "Singularities in BIEs for the Laplace equation; Joukowski trailing-edge conjecture revisited," Engineering Analysis with Boundary Elements, Vol. 25, No. 9, Oct. 2001, pp. 805 818 .

${ }^{17}$ Katz, J. and Plotkin, A., Low-Speed Aerodynamics, Cambridge University Press, 2001.

${ }^{18}$ Murua, J., Flexible Aircraft Dynamics with a Geometrically-Nonlinear Description of the Unsteady Aerodynamics, Ph.D. thesis, Imperial College London, Department of Aeronautics, May 2012.

${ }^{19}$ Simpson, R. J. S., Palacios, R., Hesse, H., and Goulart, P. J., "Predictive Control for Alleviation of Gust 
Loads on Very Flexible Aircraft," 55th AIAA/ASME/ASCE/AHS/ASC Structures, Structural Dynamics, and Materials Conference at SciTech, AIAA, National Harbour, MA, Jan. 2014.

${ }^{20}$ Simpson, R. J. S., Palacios, R., and Murua, J., "Induced Drag Calculations in the Unsteady Vortex Lattice Method," AIAA Journal, Vol. 51, No. 7, 2013, pp. 1775 - 1779.

${ }^{21}$ Simpson, R. J. S. and Palacios, R., "Numerical aspects of nonlinear flexible aircraft flight dynamics modeling," 54th AIAA/ASME/ASCE/AHS/ASC Structures, Structural Dynamics, and Materials Conference, AIAA, Boston, MA, April 2013.

${ }^{22}$ Cook, R., Palacios, R., and Goulart, P., "Robust Gust Alleviation and Stabilization of Very Flexible Aircraft," AIAA Journal, Vol. 51, No. 2, February 2013, pp. 330 - 340.

${ }^{23}$ Murua, J., Palacios, R., and Graham, J. M. R., "Applications of the unsteady vortex-lattice method in aircraft aeroelasticity and flight dynamics," Progress in Aerospace Sciences, Vol. 55, Nov. 2012, pp. 46 72 .

${ }^{24}$ Hall, K. C., "Eigenanalysis of unsteady flows about airfoils, cascades, and wings," AIAA journal, Vol. 32, 1994, pp. $2426-2432$.

${ }^{25}$ Ricketts, R. H. and Doggett, R. V., "Wind-tunnel experiments on divergence of forward-swept wings," Technical Paper TP 1685, NASA, Langley Research Center, Hampton, VA, 1980.

${ }^{26}$ Hertz, T. J., Shirk, M. H., Ricketts, R. H., and Weisshaar, T. A., "Aeroelastic tailoring with composites applied to forward swept wings," Tech. Rep. AFWAL-TR-81-3043, DTIC Document, 1981.

${ }^{27}$ Love, M. H., Zink, P. S., Wieselmann, P. A., and Youngren, H., "Body freedom flutter of high aspect ratio flying wings," The 46th AIAA/ASME/ASCE/AHS/ASC Structures, Structural Dynamics and Materials Conference, AIAA, Austin, Texas, April 2005. 


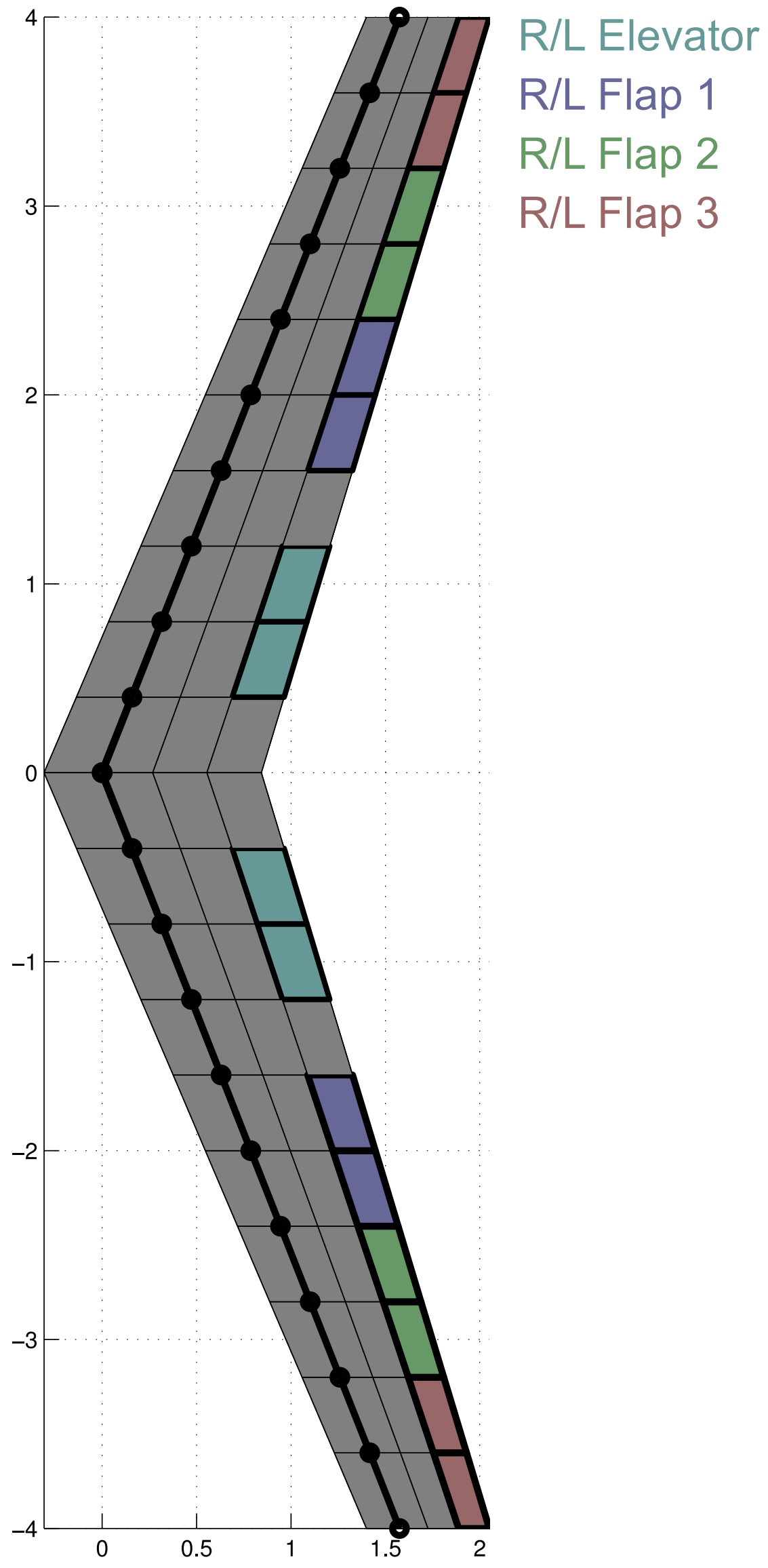

Figure 5. SFW geometry in plan view. Beam finite-elements (dotted black lines) and UVLM panels (gray/color quadrilateral lattice) corresponding to a coarse discretization. 
Mode10:5.151 Hz $\left(v_{\mathrm{z}^{2}} ; \omega_{\mathrm{z}}\right)=(-8.2735 \mathrm{e}-18,4.0133 \mathrm{e}-17,0.012104 ; 1.981 \mathrm{e}-09,0.10295,2.9532 \mathrm{e}-17)$

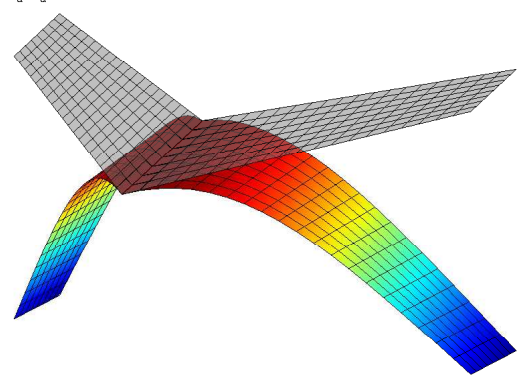

(a) Mode 10: $5.15 \mathrm{~Hz}$

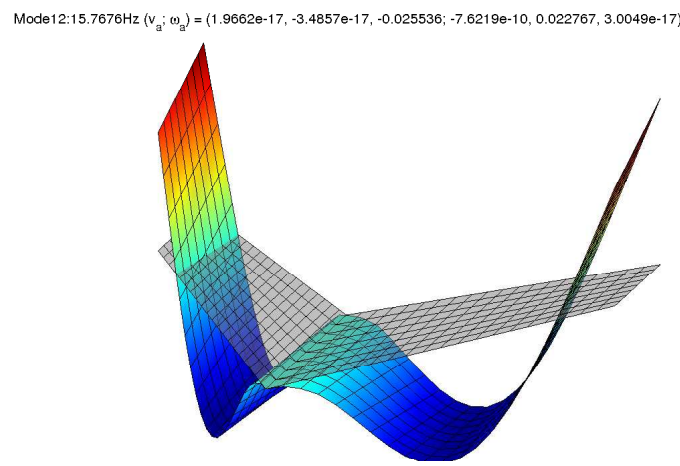

(c) Mode 12: $15.8 \mathrm{~Hz}$

Mode1 1:22.2071 Hz $\left(\mathrm{v}_{\mathrm{a}} ;\left(\mathrm{w}_{\mathrm{g}}\right)=(-0.03146,-1.8239 \mathrm{e}-09,-7.4487 \mathrm{e}-08 ;-2.0215 \mathrm{e}-09,-8.6924 \mathrm{e}-08,-4.095 \mathrm{e}-11)\right.$

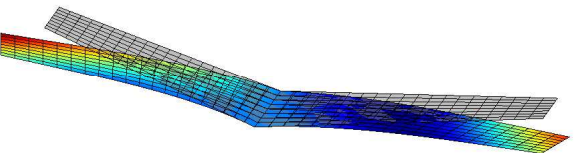

(e) Mode 14: $22.2 \mathrm{~Hz}$

Mode1 $6: 34.1995 \mathrm{~Hz}\left(\mathrm{~V}_{\mathrm{z}} ; \omega_{\mathrm{a}}\right)=(-6.6187 \mathrm{e}-18,7.3816 \mathrm{e}-18,-0.0058322 ;-6.001 \mathrm{e}-11,-0.020925,-1.1207 \mathrm{e}-18)$

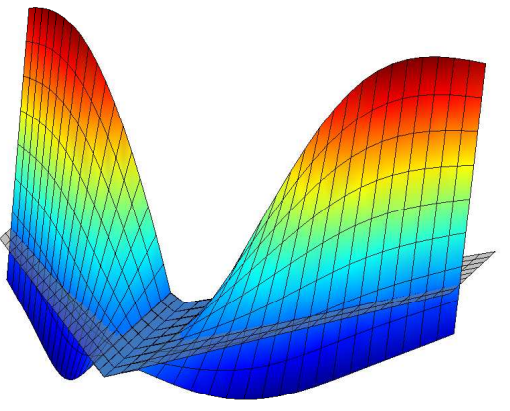

(g) Mode 16: $34.2 \mathrm{~Hz}$
Mode11:8.3079Hz $\left\langle\mathrm{v}_{\mathrm{a}^{\prime}} \omega_{\mathrm{a}}\right\}=(-3.6833 \mathrm{e}-17,-1.1652 \mathrm{e}-16,3.4644 \mathrm{e}-10 ;-0.044892,9.6502 \mathrm{e}-09,1.1254 \mathrm{e}-17)$

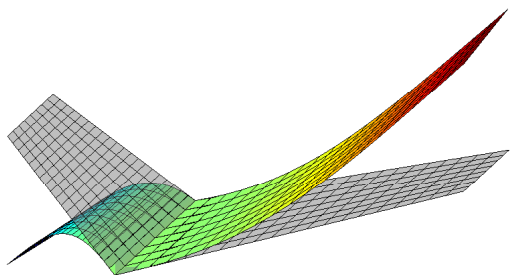

(b) Mode 11: $8.31 \mathrm{~Hz}$

Mode 13:20.31 $19 \mathrm{~Hz}\left(\mathrm{v}_{\mathrm{Z}^{\prime}} \cdot \omega_{\mathrm{z}}\right)=(1.3059 \mathrm{e}-18,-3.0064 \mathrm{e}-17,7.1067 \mathrm{e}-10 ;-0.057484,3.2058 \mathrm{e}-09,3.9673 \mathrm{e}-18)$

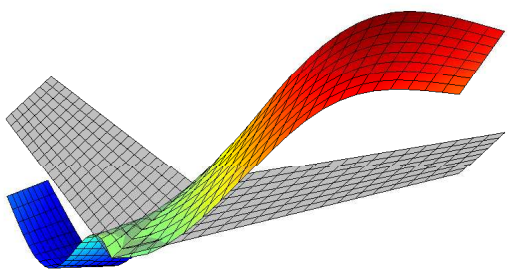

(d) Mode 13: $20.3 \mathrm{~Hz}$

Mode15:23.706 $\mathrm{Hz}\left(v_{\mathrm{z}} ; \omega_{\mathrm{z}}\right)=(1.1152 \mathrm{e}-17,-5.5405 \mathrm{e}-18,-1.0317 \mathrm{e}-10 ;-0.022696,1.8093 \mathrm{e}-09,-2.5105 \mathrm{e}-18)$

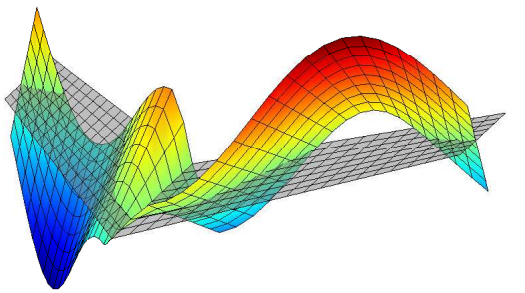

(f) Mode 15: $23.7 \mathrm{~Hz}$

Wode17:35.4285 $\mathrm{Hz}\left(\mathrm{V}_{\mathrm{z}} ; \omega_{\mathrm{a}}\right)=(3.0289 \mathrm{e}-18,-8.7218 \mathrm{e}-18,-1.7548 \mathrm{e}-10 ;-0.011398,-3.0708 \mathrm{e}-10,3.3481 \mathrm{e}-18)$

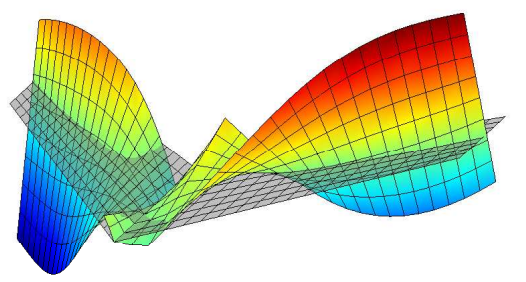

(h) Mode 17: $35.4 \mathrm{~Hz}$

Figure 6. Unconstrained structural modes of the SFW aircraft. Corresponding amplitudes of the rigid-body degrees-of-freedom are given in the figure titles; these quantities are expressed in body-fixed, fore-starboarddown axes typical of flight dynamics descriptions. 


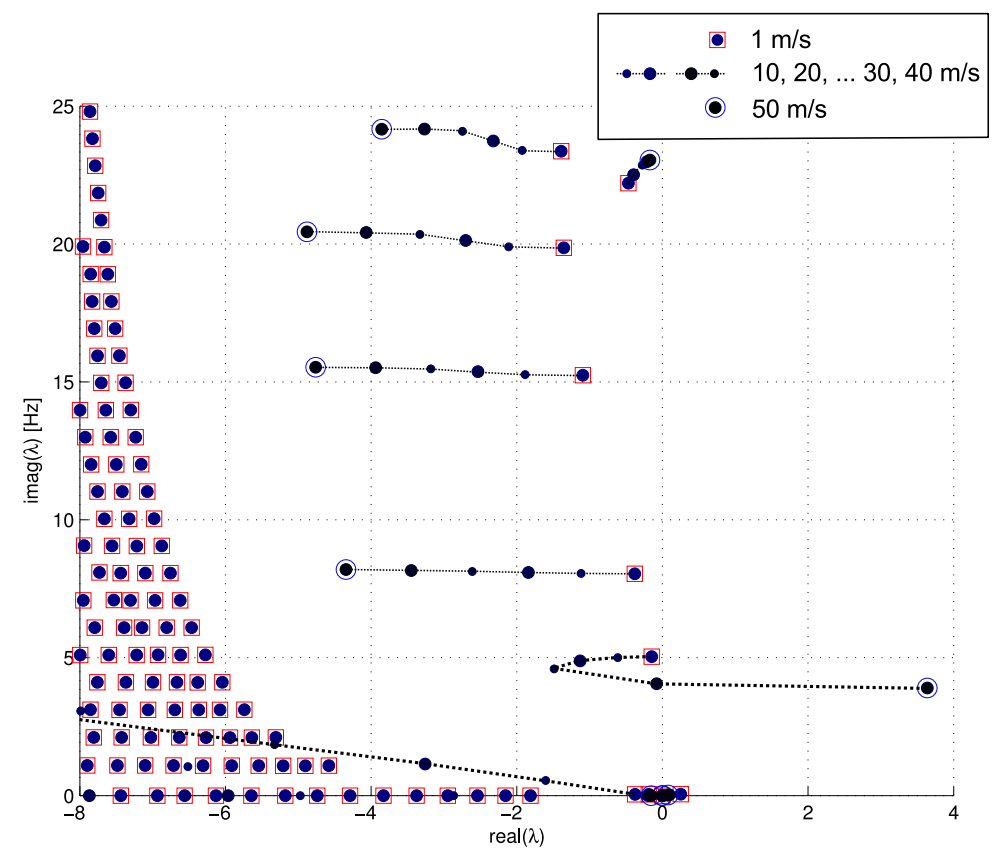

Figure 7. Aeroelastic stability plot of the unconstrained SFW aircraft at sea-level $\left(\rho_{\infty}=1.225 \mathrm{~kg} \cdot \mathbf{m}^{-3}\right)$.

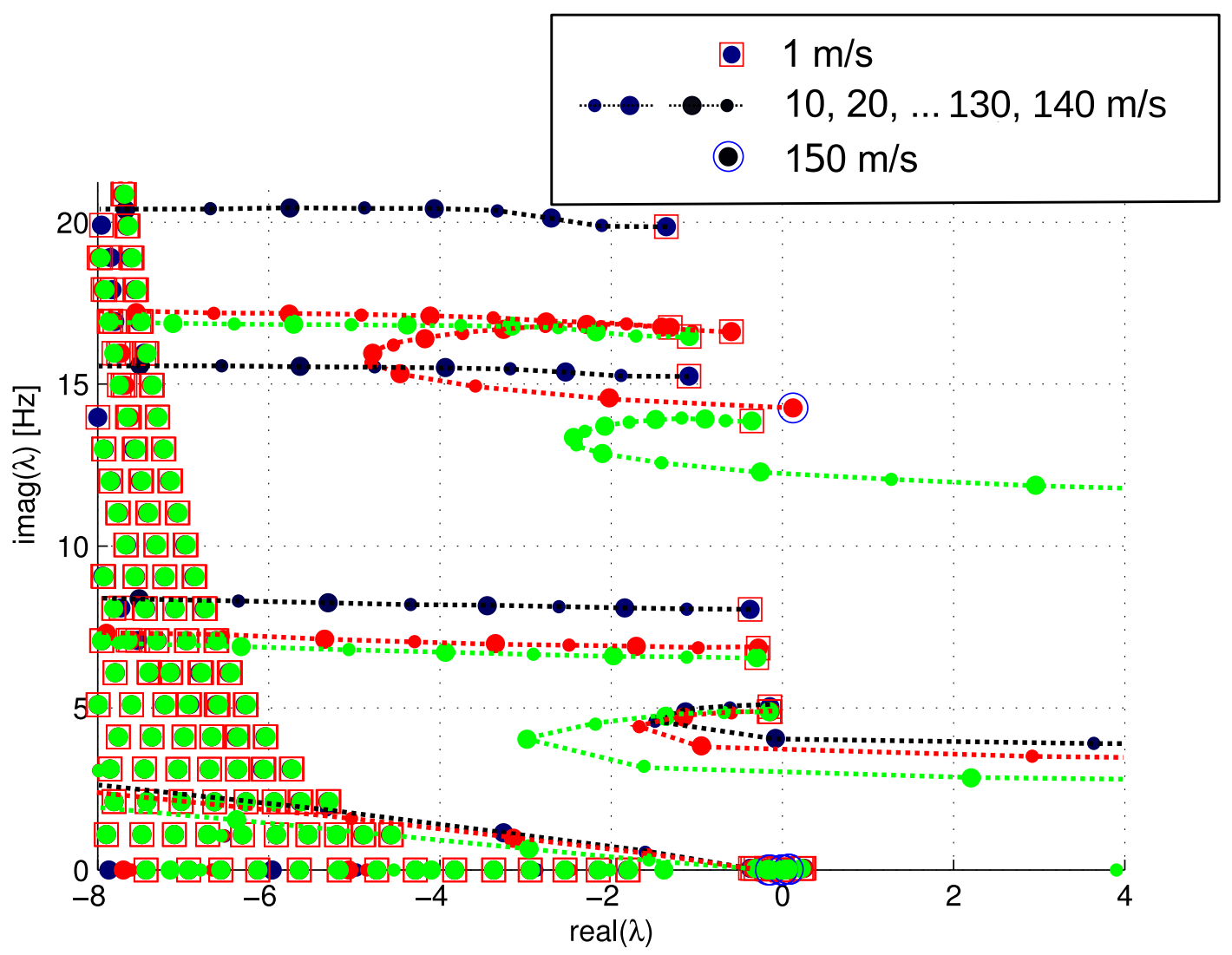

Figure 8. Aeroelastic stability plot of the unconstrained SFW aircraft with baseline mass configuration (black), lumped mass at $20 \%$ span (red), and lumped mass at $30 \%$ span (green). Sea-level altitude $\left(\rho_{\infty}=1.225\right.$ $\left.\mathrm{kg} \cdot \mathrm{m}^{-3}\right)$. 